\title{
On the Transparency of Defeasible Logics: Equivalent Premise Sets, Equivalence of Their Extensions, and Maximality of the Lower Limit*
}

\author{
Diderik Batens, Christian Straßer, and Peter Verdée ${ }^{\dagger}$ \\ Centre for Logic and Philosophy of Science \\ Ghent University, Belgium \\ \{Diderik. Batens, Christian.Strasser, Peter.Verdee @UGent . be \\ transpar.tex - June 16, 2010
}

published in Logique et Analyse, 207, 2009, pp. 281-304

\begin{abstract}
For Tarski logics, there are simple criteria that enable one to conclude that two premise sets are equivalent. We shall show that the very same criteria hold for adaptive logics, which is a major advantage in comparison to other approaches to defeasible reasoning forms.

A related property of Tarski logics is that the extensions of equivalent premise sets with the same set of formulas are equivalent premise sets. This does not hold for adaptive logics. However a very similar criterion does.

We also shall show that every monotonic logic weaker than an adaptive logic is weaker than the lower limit logic of the adaptive logic or identical to it. This highlights the role of the lower limit for settling the adaptive equivalence of extensions of equivalent premise sets.
\end{abstract}

\section{Formats for Logics for Plausible Reasoning}

This paper has a specific and a more general aim. The specific aim is related to determining whether two premise sets are equivalent with respect to logics that explicate defeasible reasoning forms - henceforth $D R F$. We shall show that adaptive logics are superior to other formats in this respect. The more general aim is to highlight the advantages of the adaptive logic program with respect to other approaches to DRF.

Let us compare the situation with Tarski logics, logics the consequence relation of which is Reflexive, Transitive and Monotonic. A variety of formulations

\footnotetext{
* Research for this paper was supported by subventions from Ghent University and from the Fund for Scientific Research - Flanders.

${ }^{\dagger}$ Peter Verdée is a post-doctoral fellow of the Fund for Scientific Research - Flanders.
} 
has been developed: axiomatic, Fitch-style, Gentzen-style, etc. Each of these have their stronger points. The variety, however, is only apparent. First, there are relatively standard procedures that, for most logics, enable one to turn one formulation into another. Next, the different formulations are at best different ways to characterize the same basic entity, viz. the consequence relation, which assigns to every premise set a consequence set. There are some differences in semantic styles as well. Again, these may be reduced to each other, except that some logics require a more complex semantics than others.

The situation is drastically different for logics that explicate DRF. Here a variety of syntactic formulations have been tried out, each of them often for some specific cases only. Many of these explications have no semantics, others require unusual techniques. ${ }^{1}$ All this raises two central questions.

A first question is whether DRF require a variety of formulations. It is indeed possible that the domain comprises reasoning forms that are so different from each other, that it is uninteresting or even impossible to forge them into the same format. Suppose, however, that it is possible to characterize all DRF by the same type of logic or logical approach. Then, presumably, there will be several such approaches. If this is so, a second question should be raised: Which are the advantages and weaknesses of the different approaches?

It is the aim of the adaptive logic program to characterize all DRF in terms of an adaptive logic in standard format (see Section 3). This was realized for a variety of DRF, mostly by tackling such reasoning forms from scratch. Many DRF have been decently described independently of the adaptive logic program. Quite a few of these were characterized by an adaptive logic in standard format - $[5,7,12,54]$ for handling inconsistent knowledge bases as in $[46,13,14]$; [10] for the signed consequence relations from $[15]$; $[18,3]$ for default reasoning and circumscription, ${ }^{2}$ documented in $[2,16,29] ;[49]$ for rational closure from [26]; [51] for abstract argumentation from [19]; [43] for the belief merging protocols from [24]. Similarly for consequence relations not described as such in the literature - $[33,32]$ for question evocation from [55]; [39, 37, 36] for abduction as described in [1]; [44, 11] for diagnosis from [45]; [35] for the notion of empirical progress from [25]. For several Tarski logics, an adaptive logic was developed to circumvent adding new premises (by tinkering) - [34] for the pragmatic structures from [40, 17]; [52] and [27] on causality as in [42]; [50, 38] for the deontic logics from [20, 21], and [53] for fuzzy logics. Those characterizations and extensions often require a translation to a different language. Where $\mathbf{L}$ is the original 'logic' and AL is an adaptive logic, the characterization may have the form: $\Gamma \vdash_{\mathbf{L}} A$ iff $f(\Gamma) \vdash_{\mathbf{A L}} f(A)$ where $f$ is a function mapping formulas from the native language (for example the standard predicative language) to a different language (for example a modal language).

The successes on the adaptive side do not entail that the first question should be answered in the negative. All that follows is that adaptive logicians were successful where one attempted to find such a characterization. The attempts were not exhaustive with respect to the present literature and new forms of defeasible logics may be discovered in the future. So the situation seems to

\footnotetext{
${ }^{1}$ With respect to the semantics, useful unifying work was done by Shoham and associates, for example [48, 23, 28]. This work is fully in line with the adaptive logic programme: there is an algorithm for turning any semantic characterization in Shoham's style into an adaptive logic.

${ }^{2}$ These are older results, not in standard format, that soon will be improved upon.
} 
justify that adaptive logicians continue their efforts, but it is possible that they will only be able to unify part of all DRF.

Let us now turn to the second question. One of the arguments adduced in favour of characterizations in terms of adaptive logics was precisely that this enterprise has a strong unifying effect, especially as the standard format provides adaptive logics with a proof theory, a semantics, and all the interesting parts of the metatheory. But obviously, unification is not the only consideration that should be taken into account.

In the present paper we shall consider a type of argument that is related to transparency. To be more precise, the argument concerns criteria for the equivalence of premise sets. This requires some explanation.

Theories may have different formulations: the same theory may be presented in different ways. To make the matter more precise, let a theory $T$ be a couple $\langle\Gamma, \mathbf{L}\rangle$, in which $\Gamma$ is a set of statements (the non-logical axioms of $T$ ) and $\mathbf{L}$ is a logic. The claims made by the theory are $C n_{\mathbf{L}}(\Gamma)=\left\{A \mid \Gamma \vdash_{\mathbf{L}} A\right\}$. That $T=$ $\langle\Gamma, \mathbf{L}\rangle$ and $T^{\prime}=\left\langle\Gamma^{\prime}, \mathbf{L}\right\rangle$ are different formulations of the same theory obviously means that $C n_{\mathbf{L}}(\Gamma)=C n_{\mathbf{L}}\left(\Gamma^{\prime}\right)$. Similarly, people talking to each other about some subject may come to the conclusion that they fully agree on the topic. If they are serious about the matter, they mean to say that all one person believes on the subject is derivable from the statements made (or agreed to) by the other. We may safely take it that the agreeing parties share the underlying logic $\mathbf{L}$, at least in the context of their present communication. So their agreement may be formally expressed by a statement of the form $C n_{\mathbf{L}}(\Gamma)=C n_{\mathbf{L}}\left(\Gamma^{\prime}\right)$. Where this statement holds true, we shall say that $\Gamma$ and $\Gamma^{\prime}$ are $\mathbf{L}$-equivalent premise sets.

Sameness of theories and mutual agreement are important matters. If two theories are the same, everything proved from one of them may be carried over immediately to the other. If two people actually agree about some subject, they are able to predict everything the other believes about the subject and they may rely on this, for example in arguments about other topics. Yet, it is obvious that offering a direct proof of $C n_{\mathbf{L}}(\Gamma)=C n_{\mathbf{L}}\left(\Gamma^{\prime}\right)$ is out of the question. Put in a more precise way, it is impossible for humans to enumerate all members of $C n_{\mathbf{L}}(\Gamma)$ and to demonstrate for each of them that it is also a member of $C n_{\mathbf{L}}\left(\Gamma^{\prime}\right) .{ }^{3}$ Humans rely on shortcuts in order to establish $C n_{\mathbf{L}}(\Gamma)=C n_{\mathbf{L}}\left(\Gamma^{\prime}\right)$.

In Section 2, we shall consider three common criteria for deciding that $C n_{\mathbf{L}}(\Gamma)=C n_{\mathbf{L}}\left(\Gamma^{\prime}\right)$. These criteria will be shown to be correct for Tarski logics. We shall show, however, that these criteria cannot be applied to certain popular formulations of DRF and that no alternatives for the criteria seem available. This will lead to the question whether there are corresponding criteria for adaptive logics. The answer is rather astonishing: the very same criteria may be applied in the case of adaptive logics. This seems a strong argument in favour of the adaptive program.

In Section 6, we shall also consider a related question. Suppose that $\Gamma$ and $\Gamma^{\prime}$ are $\mathbf{L}$-equivalent. Does it follow that $\Gamma \cup \Delta$ and $\Gamma^{\prime} \cup \Delta$ are $\mathbf{L}$-equivalent premise sets? If two people study the same theory, but possibly a different formulation of it, and both extend their formulation with the same set of statements, we might expect that the extensions are also $\mathbf{L}$-equivalent. The answer to the question will be shown to be positive for Tarski logics, but negative for most defeasible logics

\footnotetext{
${ }^{3}$ In the text, we neglect some border cases, which are irrelevant to the present discussion, for example the case in which $C n_{\mathbf{L}}(\Gamma)$ is either empty or trivial.
} 
presented in the literature. It will turn out that the answer is also negative for adaptive logics. However, in the case of adaptive logics, the answer is positive for a criterion that is extremely close to the considered one. Let $\mathbf{L}^{\prime}$ be weaker than $\mathbf{L}$ iff $C n_{\mathbf{L}^{\prime}}(\Gamma) \subset C n_{\mathbf{L}}(\Gamma)$ for some $\Gamma$ and $C n_{\mathbf{L}^{\prime}}(\Gamma) \subseteq C n_{\mathbf{L}}(\Gamma)$ for all $\Gamma$ and let $\mathbf{L}^{\prime}$ be stronger than $\mathbf{L}$ iff $\mathbf{L}$ is weaker than $\mathbf{L}^{\prime}$. The $\mathbf{L}$-equivalence of the extensions is warranted if the two premise sets are $\mathbf{L}^{\prime}$-equivalent, where $\mathbf{L}^{\prime}$ is any Tarski logic weaker than $\mathbf{L}$. We shall also present a criterion that is specific for adaptive logics and comes very handy for many premise sets.

The lower limit logic of adaptive logics is always a Tarski logic. As it is a constitutive element of the adaptive logic, it is natural to inquire whether it plays a specific role with respect to the criteria for equivalence of premise sets and for the equivalence of their extension. In Section 7, we shall show that the lower limit logic plays indeed a privileged role: if $\mathbf{L}$ is a monotonic logic and $\mathbf{L}$ is weaker than the adaptive logic or the adaptive consequence set is closed under $\mathbf{L}$, then $\mathbf{L}$ is weaker than the adaptive logic's lower limit logic or identical to it.

The conclusion will be that adaptive logics are not only attractive because of their unifying power, but also because they have certain properties which warrant a transparent handling of premise sets.

This paper does not and cannot aim at establishing a final conclusion. As we said before, new DRF may be discovered in the future. Still, the paper offers a strong argument for adaptive logics (and against some other approaches to DRF) and the argument relies on the best present insights.

\section{Equivalent Premise Sets}

Let us start with some conventions. The set of closed formulas of the considered language will be called $\mathcal{W}$. A logic $\mathbf{L}$ is a function $\mathbf{L}: \wp(\mathcal{W}) \rightarrow \wp(\mathcal{W})$, in other words a logic $\mathbf{L}$ assigns to every premise set $\Gamma$ a consequence set, which is denoted by $C n_{\mathbf{L}}(\Gamma)$. A logic is a Tarski logic iff it fulfils the following three properties:

Reflexivity: $\Gamma \subseteq C n_{\mathbf{L}}(\Gamma)$.

Transitivity: If $\Gamma^{\prime} \subseteq C n_{\mathbf{L}}(\Gamma)$ then $C n_{\mathbf{L}}\left(\Gamma^{\prime}\right) \subseteq C n_{\mathbf{L}}(\Gamma)$

Monotonicity: $C n_{\mathbf{L}}(\Gamma) \subseteq C n_{\mathbf{L}}\left(\Gamma \cup \Gamma^{\prime}\right)$.

Definition $1 \Gamma$ and $\Gamma^{\prime}$ are $\mathbf{L}$-equivalent premise sets iff $C n_{\mathbf{L}}(\Gamma)=C n_{\mathbf{L}}\left(\Gamma^{\prime}\right)$.

If $\mathbf{L}$ is a Tarski logic, three simple criteria for the $\mathbf{L}$-equivalence of premise sets are available:

C1 If $\Gamma^{\prime} \subseteq C n_{\mathbf{L}}(\Gamma)$ and $\Gamma \subseteq C n_{\mathbf{L}}\left(\Gamma^{\prime}\right)$, then $\Gamma$ and $\Gamma^{\prime}$ are $\mathbf{L}$-equivalent.

C2 If $\mathbf{L}^{\prime}$ is a Tarski logic weaker than $\mathbf{L}$, and $\Gamma$ and $\Gamma^{\prime}$ are $\mathbf{L}^{\prime}$-equivalent, then $\Gamma$ and $\Gamma^{\prime}$ are $\mathbf{L}$-equivalent.

C3 If every $C n_{\mathbf{L}}(\Delta)$ is closed under a Tarski logic $\mathbf{L}^{\prime}\left(\right.$ viz. $C n_{\mathbf{L}^{\prime}}\left(C n_{\mathbf{L}}(\Delta)\right)=$ $C n_{\mathbf{L}}(\Delta)$ for all $\left.\Delta\right)$, and $\Gamma$ and $\Gamma^{\prime}$ are $\mathbf{L}^{\prime}$-equivalent, then $\Gamma$ and $\Gamma^{\prime}$ are L-equivalent. 
Criterion C1 states that, in order for $\Gamma$ and $\Gamma^{\prime}$ to be L-equivalent, it is sufficient that all members of $\Gamma$ are $\mathbf{L}$-derivable from $\Gamma^{\prime}$ and vice versa. In terms of theories: if $T$ and $T^{\prime}$ have the same underlying logic $\mathbf{L}$ and all axioms of $T$ are $\mathbf{L}$-derivable from $T^{\prime}$ and vice versa, then the two axiom sets are $\mathbf{L}$ equivalent $-T$ and $T^{\prime}$, if different, are different formulations of the same theory. A still different rendering proceeds in terms of mutual agreement. Suppose that two persons state their views about some subject in an exhaustive way-all one of them holds true about that subject is derivable from the statements made by this party. If each party then agrees with everything the other said on the subject, one may conclude that they have the same view on the subject. C1 is an immediate consequence of the Transitivity of $\mathbf{L}$.

Criterion C2 states that if two premise sets are equivalent with respect to a Tarski logic weaker than $\mathbf{L}$, then they are equivalent with respect to $\mathbf{L}$. It is easily seen that $\mathrm{C} 2$ holds for all Tarski logics L. Suppose indeed that the antecedent of $\mathrm{C} 2$ is true. As $C n_{\mathbf{L}^{\prime}}(\Gamma) \subseteq C n_{\mathbf{L}}(\Gamma), C n_{\mathbf{L}^{\prime}}(\Gamma) \cup \Gamma \subseteq C n_{\mathbf{L}}(\Gamma)$ by the reflexivity of $\mathbf{L}$ and hence $C n_{\mathbf{L}}\left(C n_{\mathbf{L}^{\prime}}(\Gamma) \cup \Gamma\right) \subseteq C n_{\mathbf{L}}(\Gamma)$ by the transitivity of $\mathbf{L}$. So, by the monotonicity of $\mathbf{L}, C n_{\mathbf{L}}\left(C n_{\mathbf{L}^{\prime}}(\Gamma) \cup \Gamma\right)=C n_{\mathbf{L}}(\Gamma)$. Finally, as $C n_{\mathbf{L}^{\prime}}(\Gamma) \cup \Gamma=C n_{\mathbf{L}^{\prime}}(\Gamma)$ by the reflexivity of $\mathbf{L}^{\prime}, C n_{\mathbf{L}}\left(C n_{\mathbf{L}^{\prime}}(\Gamma)\right)=C n_{\mathbf{L}}(\Gamma)$. By the same reasoning $C n_{\mathbf{L}}\left(C n_{\mathbf{L}^{\prime}}\left(\Gamma^{\prime}\right)\right)=C n_{\mathbf{L}}\left(\Gamma^{\prime}\right)$. As $C n_{\mathbf{L}^{\prime}}(\Gamma)=C n_{\mathbf{L}^{\prime}}\left(\Gamma^{\prime}\right)$, $C n_{\mathbf{L}}(\Gamma)=C n_{\mathbf{L}}\left(\Gamma^{\prime}\right)$.

Criterion C3 is related to the fact that we expect operations under which $\mathbf{L}$-consequence sets are closed to define a logic that is weaker than $\mathbf{L}$ or identical to it, which triggers C2. If, for all $\Delta, A \wedge B \in C n_{\mathbf{L}}(\Delta)$ just in case $A \in C n_{\mathbf{L}}(\Delta)$ and $B \in C n_{\mathbf{L}}(\Delta)$, then we expect $\Gamma \cup\{p \wedge q\}$ and $\Gamma \cup\{p, q\}$ to be $\mathbf{L}$-equivalent premise sets.

Incidently, that $\mathbf{L}^{\prime}$ is a Tarski logic is essential for both $\mathrm{C} 2$ and C3. If $\mathbf{L}^{\prime}$ were an arbitrary logic, these criteria would not hold. To see this, let $\mathcal{W}$ be the set of closed formulas of the standard language, let $\mathbf{C L}$ be classical logic and let $\mathbf{L}^{\prime}$ be defined by $C n_{\mathbf{L}^{\prime}}(\Gamma)=\left\{A \in \Gamma \mid\right.$ for all $B \in \mathcal{W}, B \notin C n_{\mathbf{C L}}(\{A\})$ or $B \in \Gamma\}$. In words, the $\mathbf{L}^{\prime}$-consequence set of $\Gamma$ are those members of $\Gamma$ of which all CL-consequences are members of $\Gamma$. Obviously, it holds for all $\Delta$ that $C n_{\mathbf{L}^{\prime}}(\Delta) \subseteq C n_{\mathbf{C L}}(\Delta)$ and also that $C n_{\mathbf{L}^{\prime}}\left(C n_{\mathbf{C L}}(\Delta)\right)=C n_{\mathbf{C L}}(\Delta)$. However, there are infinitely many $\Gamma$ for which no $A \in \Gamma$ is such that $C n_{\mathbf{C L}}(A) \subseteq \Gamma$. For all of them $C n_{\mathbf{L}^{\prime}}(\Gamma)=C n_{\mathbf{L}^{\prime}}(\emptyset)$ but $C n_{\mathbf{C L}}(\Gamma) \neq C n_{\mathbf{C L}}(\emptyset)$.

Obviously, C1 may be combined with C2 or C3. Thus if $\mathbf{L}^{\prime}$ is a Tarski logic weaker than $\mathbf{L}, \Gamma^{\prime} \subseteq C n_{\mathbf{L}^{\prime}}(\Gamma)$ and $\Gamma \subseteq C n_{\mathbf{L}^{\prime}}\left(\Gamma^{\prime}\right)$, then $\Gamma$ and $\Gamma^{\prime}$ are $\mathbf{L}$-equivalent.

Let us now turn to defeasible logics. Consider first the Strong (also called Inevitable) and Weak consequence relations from [46] - see also [13]. Given a possibly inconsistent set of premises $\Gamma, \Delta \subseteq \Gamma$ is a maximal consistent subset of $\Gamma$ iff, for all $A \in \Gamma-\Delta, \Delta \cup\{A\}$ is inconsistent. $\Gamma \vdash_{\text {Strong }} A$ iff $A$ is a CL-consequence of every maximal consistent subset of $\Gamma$ and $\Gamma \vdash_{W e a k} A$ iff $A$ is a CL-consequence of some maximal consistent subset of $\Gamma$.

It is easily seen that $\mathrm{C} 1$ does not hold for the Weak consequence relation. Here is an example: $\{p, q, \neg p\} \subseteq C n_{W e a k}(\{p \wedge q, \neg p\})$ and $\{p \wedge q, \neg p\} \subseteq$ $C n_{\text {Weak }}(\{p, q, \neg p\})$, but $\neg p \wedge q \in C n_{W e a k}(\{p, q, \neg p\})$ whereas $\neg p \wedge q \notin$ $C n_{W e a k}(\{p \wedge q, \neg p\})$. It is also easily seen that C3 does not hold for the Strong consequence relation. Let $\mathbf{L C}$ be the Tarski logic that consists, apart from the Premise rule, of the rules Adjunction and Simplification. All Strong consequence sets are closed under $\mathbf{L C}$, viz. $C n_{\text {Strong }}(\Gamma)=C n_{\mathbf{L C}}\left(C n_{\text {Strong }}(\Gamma)\right)$ for all $\Gamma$. However, $C n_{\mathbf{L C}}(\{p, q, \neg p\})=C n_{\mathbf{L C}}(\{p \wedge q, \neg p\})$ but $C n_{\text {Strong }}(\{p, q, \neg p\}) \neq$ 
$C n_{\text {Strong }}(\{p \wedge q, \neg p\})$, for example $p, q, \neg p \vdash_{\text {Strong }} q$ whereas $p \wedge q, \neg p \nvdash_{\text {Strong }} q$.

For an example of a logic for which $\mathrm{C} 2$ does not hold, we shall remain close to the Rescher-Manor consequence relations, adding a (weak) Schotch-Jennings flavour - see for example [47]. A partition of $\Gamma$ is a set of sets $\left\{\Gamma_{1}, \ldots, \Gamma_{n}\right\}$ $(n \geq 1)$ such that $\Gamma=\Gamma_{1} \cup \ldots \cup \Gamma_{n}$ and $\Gamma_{i} \cap \Gamma_{j}=\emptyset$ for all different $i, j \in$ $\{1, \ldots, n\}$. A partition $\left\{\Gamma_{1}, \ldots, \Gamma_{n}\right\}$ of $\Gamma$ is consistent iff every $\Gamma_{i}(1 \leq i \leq n)$ is consistent. Obviously, $\Gamma$ has a consistent partition iff all $A \in \Gamma$ are consistent. The regular partitions of $\Gamma$ are the consistent ones or, if there are no consistent ones, all partitions of $\Gamma$. Define: $A \in C n_{\mathbf{R}}(\Gamma)$ iff there is a regular partition $\left\{\Gamma_{1}, \ldots, \Gamma_{n}\right\}$ of $\Gamma$ and an $i(1 \leq i \leq n)$ such that $A \in C n_{\mathbf{C L}}\left(\Gamma_{i}\right)$. Define $C n_{\mathbf{Q}}(\Gamma)=C n_{\mathbf{P}}\left(C n_{\mathbf{R}}(\Gamma)\right)$, in which $\mathbf{P}$ is full positive $\mathbf{C L}$. If $\{\Gamma\}$ is a regular partition of $\Gamma, C n_{\mathbf{Q}}(\Gamma)=C n_{\mathbf{C L}}(\Gamma)$; if some $A \in \Gamma$ is inconsistent, $C n_{\mathbf{Q}}(\Gamma)$ is trivial; if $\Gamma$ is inconsistent but all $A \in \Gamma$ are consistent, $C n_{\mathbf{Q}}(\Gamma)$ is inconsistent but non-trivial, border cases aside. Note that $\mathbf{P}$ is a Tarski logic and that it is weaker than $\mathbf{Q}$, viz. $C n_{\mathbf{P}}(\Gamma) \subseteq C n_{\mathbf{Q}}(\Gamma)$ for all $\Gamma$.

C2 does not hold for the defeasible logic $\mathbf{Q}$. Indeed, $\mathbf{P}$ is a Tarski logic weaker than $\mathbf{Q}$ and $C n_{\mathbf{P}}(\{p, \neg p\})=C n_{\mathbf{P}}(\{p \wedge \neg p\})$, but $C n_{\mathbf{Q}}(\{p \wedge \neg p\})$ is trivial whereas $C n_{\mathbf{Q}}(\{p, \neg p\})$ is not.

These examples are rather 'generous' because the situation is actually worse for certain systems describing DRF. For example for the many kinds of default logics the criteria $\mathrm{C} 1-3$ should be reformulated in order to make a chance to be applicable. The set of defaults has to enter the picture and 'facts' and defaults are to some extend exchangeable. The situation is similar for many other logics that characterize DFR, even for the very transparent pivotal-assumption consequences defined in [31].

\section{A Brief Introduction to the Standard Format}

Adaptive logics adapt themselves to the premise set they are applied to. The logic adapts itself: it depends on the premise set whether a specific application of an inference rule is or is not correct. The present most attractive description of adaptive logics is called the standard format, appearing from [6] on and most extensively studied in [8], to which we refer for details and metatheoretic proofs. Nearly all known adaptive logics have been phrased in standard format.

An adaptive logic $\mathbf{A L}$ is defined by a triple:

1. A lower limit logic LLL: a reflexive, transitive, monotonic, and compact logic that has a characteristic semantics and contains CL (Classical Logic).

2. A set of abnormalities $\Omega$ : a set of $\mathbf{L L L}$-contingent formulas, characterized by a (possibly restricted) logical form $\mathrm{F}$ which contains at least one logical symbol.

3. An adaptive strategy: Reliability or Minimal Abnormality.

The lower limit logic is the stable part of the adaptive logic; anything that follows from the premises by LLL will never be revoked. The lower limit logic is an extension of $\mathbf{C L}$ because it contains all the classical symbols (noted as $\check{\neg}, \check{V}, \check{\wedge}, \check{\supset}, \check{\exists}, \check{\forall}$, and $\check{=}$ ) next to its standard symbols (noted without check). ${ }^{4}$ In

\footnotetext{
${ }^{4}$ Actually, we only use the checked symbol where the corresponding symbol of the standard language has a different meaning than the CL-symbol.
} 
standard applications, the classical symbols do not occur in the premises or in the conclusion. Their function is technical and metatheoretical. Abnormalities are supposed to be false, 'unless and until proven otherwise'. Strategies are ways to cope with derivable disjunctions of abnormalities: an adaptive strategy picks one specific way to interpret the premises as normally as possible. ${ }^{5}$

Two typical examples of adaptive logics are the inconsistency-adaptive logics $\mathbf{C L u N}^{r}$ and $\mathbf{C L u N}{ }^{m}$. They are defined as follows. The lower limit logic is CLuN ( $C$ lassical Logic allowing for gl $u$ ts with respect to $N$ egation), viz. full positive CL with $(A \supset \neg A) \supset \neg A$ added as the only axiom for the standard negation, and extended with classical negation $\check{\neg} .{ }^{6}$ While $A \vee \neg A$ is a CLuNtheorem, $A \wedge \neg A$ is $\mathbf{C L u N - c o n t i n g e n t . ~ T h e ~ s e t ~ o f ~ a b n o r m a l i t i e s ~} \Omega$ comprises all formulas of the form $\exists(A \wedge \neg A)$ (the existential closure of $A \wedge \neg A)$. The strategies are respectively Reliability and Minimal Abnormality - see below. The resulting adaptive logics will be called $\mathbf{C L u N} \mathbf{N}^{r}$ and $\mathbf{C L u N ^ { m }}$.

Incidentally, if the lower limit logic is extended with an axiom that declares all abnormalities logically false, one obtains the upper limit logic ULL. If a premise set $\Gamma$ does not require that any abnormalities are true, the $\mathbf{A L}$ consequences of $\Gamma$ are identical to its ULL-consequences. The upper limit logic of $\mathbf{C L u N} \mathbf{N}^{r}$ and of $\mathbf{C L u N} \mathbf{N}^{m}$ is $\mathbf{C L}$.

In the expression $\operatorname{Dab}(\Delta), \Delta$ is a finite subset of $\Omega$ and $\operatorname{Dab}(\Delta)$ denotes the classical disjunction of the members of $\Delta . \operatorname{Dab}(\Delta)$ is called a $D a b$-formula. $\operatorname{Dab}(\Delta)$ is a minimal Dab-consequence of $\Gamma$ iff $\Gamma \vdash_{\mathbf{L L L}} \operatorname{Dab}(\Delta)$ whereas $\Gamma \nvdash_{\mathbf{L L L}}$ $\operatorname{Dab}\left(\Delta^{\prime}\right)$ for any $\Delta^{\prime} \subset \Delta$. Where $\operatorname{Dab}\left(\Delta_{1}\right), \operatorname{Dab}\left(\Delta_{2}\right), \ldots$ are the minimal Dab-consequences of $\Gamma, U(\Gamma)=\Delta_{1} \cup \Delta_{2} \cup \ldots$ The set $U(\Gamma)$ comprises the abnormalities that are unreliable with respect to $\Gamma$. Where $M$ is a LLL-model, $A b(M)$ is the set of abnormalities verified by $M$.

Definition 2 A LLL-model $M$ of $\Gamma$ is reliable iff $A b(M) \subseteq U(\Gamma)$.

Definition $3 \Gamma \vDash_{\mathbf{A L}^{r}} A$ iff $A$ is verified by all reliable models of $\Gamma$.

Definition $4 A$ LLL-model $M$ of $\Gamma$ is minimally abnormal iff there is no LLLmodel $M^{\prime}$ of $\Gamma$ such that $A b\left(M^{\prime}\right) \subset A b(M)$.

Definition $5 \Gamma \vDash_{\mathbf{A L}^{m}} A$ iff $A$ is verified by all minimally abnormal models of $\Gamma$.

The two strategies are not as different as the above treatment may suggest. A choice set of $\Sigma=\left\{\Delta_{1}, \Delta_{2}, \ldots\right\}$ is a set that contains an element out of each member of $\Sigma$. A minimal choice set of $\Sigma$ is a choice set of $\Sigma$ of which no proper subset is a choice set of $\Sigma$. Where $\operatorname{Dab}\left(\Delta_{1}\right), \operatorname{Dab}\left(\Delta_{2}\right), \ldots$ are the minimal $D a b$ consequences of $\Gamma, \Phi(\Gamma)$ is the set of minimal choice sets of $\Sigma=\left\{\Delta_{1}, \Delta_{2}, \ldots\right\}$. It can be shown that a LLL-model $M$ of $\Gamma$ is minimally abnormal iff $A b(M) \in$ $\Phi(\Gamma)$.

Adaptive logics have not only a semantics, but also a dynamic proof theorysee for example [9] for some theory. An annotated AL-proof consists of lines that

\footnotetext{
${ }^{5}$ Apart from Reliability and Minimal Abnormality, several strategies were developed mainly in order to characterize consequence relations from the literature in terms of an adaptive logic. All those strategies can be reduced to Reliability or Minimal Abnormality under a translation.

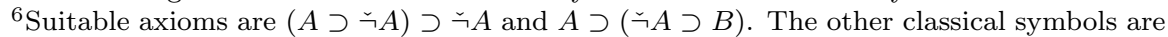
stipulated to be identical to the corresponding standard symbols.
} 
have four elements: a line number, a formula, a justification and a condition. Where

\section{$A \quad \Delta$}

abbreviates that $A$ occurs in the proof as the formula of a line that has $\Delta$ as its condition, the (generic) inference rules are:

PREM If $A \in \Gamma$ :

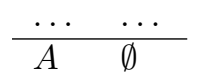

RU

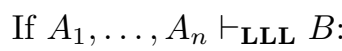

$A_{1} \quad \Delta_{1}$

. . . .

\begin{tabular}{ll}
$A_{n}$ & $\Delta_{n}$ \\
\hline$B$ & $\Delta_{1} \cup \ldots \cup \Delta_{n}$
\end{tabular}

$\mathrm{RC}$

$$
\text { If } \begin{array}{rll}
A_{1}, \ldots, A_{n} \vdash_{\mathbf{L L L}} B \check{V} \operatorname{Dab}(\Theta) & A_{1} & \Delta_{1} \\
& \ldots & \ldots \\
& A_{n} & \Delta_{n} \\
\hline & B & \Delta_{1} \cup \ldots \cup \Delta_{n} \cup \Theta
\end{array}
$$

We shall need to consider stages of proofs, which are lists of lines obtained by applications of the three above rules, with the usual understanding that the justification of a line should only refer to lines preceding it in the list. The empty list will be considered as stage 0 of every proof. Where $s$ is a stage, $\mathrm{s}^{\prime}$ is an extension of $\mathrm{s}$ iff all lines that occur in $\mathrm{s}$ occur in the same order in $\mathrm{s}^{\prime}$. A (dynamic) proof is a chain of stages. Here comes a peculiarity required by the Minimal Abnormality strategy. Normally, the extension of a stage is obtained by appending lines. This is not required here. The added lines may be inserted, provided that the justification of every line refers only to preceding lines. A line inserted between lines 4 and 5 may, for example, be numbered 4.1. ${ }^{7}$

That $A$ is derivable on the condition $\Delta$ may be interpreted as follows: it follows from the premise set that $A$ or one of the members of $\Delta$ is true. As the members of $\Delta$, which are abnormalities, are supposed to be false, $A$ is considered as derived, unless and until it shows that the supposition cannot be upheld. The precise meaning of "cannot be upheld" depends on the strategy, which determines the marking definition (see below) and hence determines which lines are marked at a stage. If a line is marked at a stage, its formula is considered as not derived at that stage.

We now set out to present the marking definitions. $\operatorname{Dab}(\Delta)$ is a minimal Dab-formula at stage s of an AL-proof iff $\operatorname{Dab}(\Delta)$ has been derived at that stage on the condition $\emptyset$ whereas there is no $\Delta^{\prime} \subset \Delta$ for which $\operatorname{Dab}\left(\Delta^{\prime}\right)$ has been derived on the condition $\emptyset$.

Consider a proof from $\Gamma$ at stage s and let $\operatorname{Dab}\left(\Delta_{1}\right), \ldots, \operatorname{Dab}\left(\Delta_{n}\right)$ be the minimal $D a b$-formulas at that stage. $U_{s}(\Gamma)=\Delta_{1} \cup \ldots \cup \Delta_{n}$ and $\Phi_{s}(\Gamma)$ is the set of minimal choice sets of $\left\{\Delta_{1}, \ldots, \Delta_{n}\right\}$.

Definition 6 Marking for Reliability: Line $l$ is marked at stage s iff, where $\Delta$ is its condition, $\Delta \cap U_{s}(\Gamma) \neq \emptyset$.

\footnotetext{
${ }^{7}$ An alternative, which we shall not consider in this paper, is to renumber all lines after the insertion and to adjust the old line numbers in the justifications.
} 
Definition 7 Marking for Minimal Abnormality: Line l is marked at stage s iff, where $A$ is derived on the condition $\Delta$ on line $l$, (i) there is no $\varphi \in \Phi_{s}(\Gamma)$ such that $\varphi \cap \Delta=\emptyset$, or (ii) for some $\varphi \in \Phi_{s}(\Gamma)$, there is no line on which $A$ is derived on a condition $\Theta$ for which $\varphi \cap \Theta=\emptyset$.

This reads more easily: where $A$ is derived on the condition $\Delta$ on line $l$, line $l$ is unmarked at stage $\mathrm{s}$ iff (i) there is a $\varphi \in \Phi_{s}(\Gamma)$ for which $\varphi \cap \Delta=\emptyset$ and (ii) for every $\varphi \in \Phi_{s}(\Gamma)$, there is a line at which $A$ is derived on a condition $\Theta$ for which $\varphi \cap \Theta=\emptyset$.

A formula $A$ is derived at stage s of a proof from $\Gamma$ iff it is the formula of a line that is unmarked at that stage. Marks may come and go as the proof proceeds. So one also wants to define a stable notion of derivability, which is called final derivability.

Definition $8 A$ is finally derived from $\Gamma$ on line $l$ of a stage $\mathrm{s}$ iff (i) $A$ is the second element of line $l$, (ii) line $l$ is not marked at stage s, and (iii) every extension of the stage in which line $l$ is marked may be further extended in such a way that line $l$ is unmarked.

Definition $9 \Gamma \vdash_{\mathbf{A L}} A$ ( $A$ is finally $\mathbf{A L}$-derivable from $\Gamma$ ) iff $A$ is finally derived on a line of a proof from $\Gamma$.

In Definition 8, s may be taken to be a finite stage for both strategies. For the Reliability strategy, the definition may moreover be taken to refer to finite extensions only. For Minimal Abnormality the definition should be required to refer to finite as well as to infinite extensions, as was shown in [4, p. 479].

The intuitive notion behind final derivability is the existence of a proof that is stable with respect to an unmarked line $l: A$ is derived on line $l$ and line $l$ is unmarked in the proof and in all its extensions. However, for some $\mathbf{A L}, \Gamma$, and $A$, only an infinite proof from $\Gamma$ in which $A$ is the formula of a line $l$ is stable with respect to line $l$.

Definition 8 has an attractive game-theoretic interpretation - see especially [9]. The proponent has shown that $A$ is finally derived on line $l$ iff, whenever the opponent extends the proof in such a way that line $l$ is marked, the proponent is able to extend the extension further in such a way that line $l$ is unmarked. Note that the preceding sentence refers to all possible extensions. So it can only be established by a reasoning in the metalanguage.

Before closing this section, we list, for future reference, some theorems that are proven in [8]. The original numbers are mentioned in brackets.

Theorem $1 \Gamma \subseteq C n_{\mathbf{A L}}(\Gamma)$. (Reflexivity) [Th. 11.2]

Theorem $2 C n_{\mathbf{A L}}\left(C n_{\mathbf{A L}}(\Gamma)\right)=C n_{\mathbf{A L}}(\Gamma)$. (Fixed Point) [Th. 11.6-7]

Theorem 3 If $\Gamma^{\prime} \subseteq C n_{\mathbf{A L}}(\Gamma)$ then $C n_{\mathbf{A L}}(\Gamma)=C n_{\mathbf{A L}}\left(\Gamma \cup \Gamma^{\prime}\right)$. [Th. 11.10]

Note that the Theorem 3 is an immediate consequence of the following two

- Cautious Monotonicity (if $\Gamma^{\prime} \subseteq C n_{\mathbf{A L}}(\Gamma)$ then $C n_{\mathbf{A L}}(\Gamma) \subseteq C n_{\mathbf{A L}}\left(\Gamma \cup \Gamma^{\prime}\right)$ ) [Th. 11.12]

- Cumulative Transitivity (if $\Gamma^{\prime} \subseteq C n_{\mathbf{A L}}(\Gamma)$ then $C n_{\mathbf{A L}}\left(\Gamma \cup \Gamma^{\prime}\right) \subseteq C n_{\mathbf{A L}}(\Gamma)$ ) [Th. 11.11]. 
Theorem $4 \Gamma \vdash \mathbf{A L}^{m}$ A iff, for every $\varphi \in \Phi(\Gamma)$, there is a $\Delta \subset \Omega$ such that $\Delta \cap \varphi=\emptyset$ and $\Gamma \vdash_{\text {LLL }} A \breve{V} \operatorname{Dab}(\Delta)$. [Th. 8]

Theorem $5 C n_{\mathbf{L L L}}(\Gamma) \subseteq C n_{\mathbf{A L}^{r}}(\Gamma) \subseteq C n_{\mathbf{A L}^{m}}(\Gamma) \subseteq C n_{\mathbf{U L L}}(\Gamma)$. [Th. 11.1]

From here on, "adaptive logic" will always refer to an adaptive logic in standard format.

\section{Equivalent Premise Sets and Adaptive Logics}

It was proved that all adaptive logics have the properties Reflexivity, Cumulative Transitivity, Cautious Monotonicity, and Fixed Point. From this it is easily provable that $\mathrm{C} 1-\mathrm{C} 3$ hold for all of them. Note that each of the three criteria greatly simplifies the identification of equivalent premise sets (or theories).

Theorem 6 C1 holds for all adaptive logics.

Proof. Suppose that $\Gamma^{\prime} \subseteq C n_{\mathbf{A L}}(\Gamma)$ and $\Gamma \subseteq C n_{\mathbf{A L}}\left(\Gamma^{\prime}\right)$. By Theorem 3, $C n_{\mathbf{A L}}(\Gamma)=C n_{\mathbf{A L}}\left(\Gamma \cup \Gamma^{\prime}\right)$ and $C n_{\mathbf{A L}}\left(\Gamma^{\prime}\right)=C n_{\mathbf{A L}}\left(\Gamma \cup \Gamma^{\prime}\right)$. So $C n_{\mathbf{A L}}(\Gamma)=$ $C n_{\mathrm{AL}}\left(\Gamma^{\prime}\right)$.

The following lemma establishes that $\mathrm{C} 2$ and $\mathrm{C} 3$ are coextensive whenever $\mathbf{L}$ is reflexive and $C n_{\mathbf{L}}(\Gamma)$ is a fixed point.

Lemma 1 If $\mathbf{L}$ is reflexive, $C n_{\mathbf{L}}(\Gamma)$ is a fixed point $\left(C n_{\mathbf{L}}(\Gamma)=C n_{\mathbf{L}}\left(C n_{\mathbf{L}}(\Gamma)\right)\right.$ for all $\Gamma)$, and $\mathbf{L}^{\prime}$ is reflexive and monotonic, then $C n_{\mathbf{L}^{\prime}}\left(C n_{\mathbf{L}}(\Gamma)\right)=C n_{\mathbf{L}}(\Gamma)$ for all $\Gamma$ iff $C n_{\mathbf{L}^{\prime}}(\Gamma) \subseteq C n_{\mathbf{L}}(\Gamma)$ for all $\Gamma . \quad$ (Closure Lemma)

Proof. Suppose that the antecedent is true. So, for all $\Gamma, \Gamma \subseteq C n_{\mathbf{L}}(\Gamma)$ by the reflexivity of $\mathbf{L}$ and hence, for all $\Gamma, C n_{\mathbf{L}^{\prime}}(\Gamma) \subseteq C n_{\mathbf{L}^{\prime}}\left(C n_{\mathbf{L}}(\Gamma)\right)$ by the monotonicity of $\mathbf{L}^{\prime}$. We have to prove an equivalence.

$\Rightarrow$ Suppose that, for all $\Gamma, C n_{\mathbf{L}^{\prime}}\left(C n_{\mathbf{L}}(\Gamma)\right)=C n_{\mathbf{L}}(\Gamma) . \Gamma \subseteq C n_{\mathbf{L}}(\Gamma)$ by the reflexivity of $\mathbf{L}$. So $C n_{\mathbf{L}^{\prime}}(\Gamma) \subseteq C n_{\mathbf{L}^{\prime}}\left(C n_{\mathbf{L}}(\Gamma)\right)$ by the monotonicity of $\mathbf{L}^{\prime}$. From this and the supposition follows that, for all $\Gamma, C n_{\mathbf{L}^{\prime}}(\Gamma) \subseteq C n_{\mathbf{L}}(\Gamma)$.

$\Leftarrow$ Suppose that, for all $\Gamma, C n_{\mathbf{L}^{\prime}}(\Gamma) \subseteq C n_{\mathbf{L}}(\Gamma)$ and hence $C n_{\mathbf{L}^{\prime}}\left(C n_{\mathbf{L}}(\Gamma)\right) \subseteq$ $C n_{\mathbf{L}}\left(C n_{\mathbf{L}}(\Gamma)\right)$. As $C n_{\mathbf{L}}(\Gamma)$ is a fixed point, it follows that $C n_{\mathbf{L}^{\prime}}\left(C n_{\mathbf{L}}(\Gamma)\right) \subseteq$ $C n_{\mathbf{L}}(\Gamma)$ for all $\Gamma$. So, by the reflexivity of $\mathbf{L}^{\prime}, C n_{\mathbf{L}^{\prime}}\left(C n_{\mathbf{L}}(\Gamma)\right)=C n_{\mathbf{L}}(\Gamma)$ for all $\Gamma$.

Theorem 7 C2 and C3 hold for all adaptive logics.

Proof. C2 and C3 are coextensive for all adaptive logics because of Lemma 1 together with Theorems 1 and 2. It suffices to prove that $\mathrm{C} 2$ holds for all adaptive logics. Suppose that the antecedent of $\mathrm{C} 2, C n_{\mathbf{L}^{\prime}}(\Gamma) \subseteq C n_{\mathbf{A L}}(\Gamma)$, holds true for all $\Gamma$. As $\mathbf{A L}$ is reflexive (Theorem 1), it follows that

$$
\Gamma \cup C n_{\mathbf{L}^{\prime}}(\Gamma) \subseteq C n_{\mathbf{A L}}(\Gamma) .
$$

From this, by Theorem 3,

$$
C n_{\mathbf{A L}}(\Gamma)=C n_{\mathbf{A L}}\left(\Gamma \cup C n_{\mathbf{L}^{\prime}}(\Gamma)\right),
$$


whence, as $\mathbf{L}^{\prime}$ is reflexive,

$$
C n_{\mathbf{A L}}(\Gamma)=C n_{\mathbf{A L}}\left(C n_{\mathbf{L}^{\prime}}(\Gamma)\right) .
$$

By the same reasoning

$$
C n_{\mathbf{A L}}\left(\Gamma^{\prime}\right)=C n_{\mathbf{A L}}\left(C n_{\mathbf{L}^{\prime}}\left(\Gamma^{\prime}\right)\right) .
$$

So, as $C n_{\mathbf{L}^{\prime}}(\Gamma)=C n_{\mathbf{L}^{\prime}}\left(\Gamma^{\prime}\right)$ by the supposition,

$$
C n_{\mathbf{A L}}(\Gamma)=C n_{\mathbf{A L}}\left(\Gamma^{\prime}\right) .
$$

Note that, for every adaptive logic $\mathbf{A L}, \mathbf{L L L}$ is a Tarski logic weaker than AL. So if two premise sets are LLL-equivalent, they are also $\mathbf{A L}$-equivalent in view of $\mathrm{C} 2$. For some premise sets, however, one needs to rely directly on C1. An example is that $C n_{\mathbf{C L u N}^{m}}(\{p\})=C n_{\mathbf{C L u N}^{m}}(\{p \vee(q \wedge \neg q)\})$. While $C n_{\mathrm{CLuN}}(\{p\}) \neq C n_{\mathrm{CLuN}}(\{p \vee(q \wedge \neg q)\})$, it is easy enough to show that $\{p\} \vdash_{\mathbf{C L u N}^{m}} p \vee(q \wedge \neg q)$ and that $\{p \vee(q \wedge \neg q)\} \vdash_{\mathbf{C L u N}^{m}} p$.

\section{Characterizations Under a Translation}

Some readers may be puzzled by our claim that criteria C1-3 hold for the characterization of a DRF in terms of an adaptive logic while they do not hold for other characterizations - see Section 1. The reason is that the former characterizations are realized under a translation. In order to make this paper more self-contained, we present two examples. Consider first the logic $\mathbf{Q}$ from Section 2, restricting the discussion to the propositional level.

Let $\triangleright$ abbreviate $\square \diamond$. Where $\mathbf{T}$ is the well-known modal logic of Feys - see, for example, $[22]^{8}$ - let the modal logic $\mathbf{T}^{m}$ be defined by (i) the lower limit $\mathbf{T}$, (ii) the set of abnormalities $\Omega=\{(\triangleright A \wedge \triangleright B) \wedge \neg \triangleright(A \wedge B) \mid A, B \in \mathcal{W}\}$, and (iii) Minimal Abnormality. It is provable that, where $B_{1} \wedge \ldots \wedge B_{n}$ is the conjunctive normal form of $A$ and $\Gamma^{\triangleright}=\{\triangleright C \mid C \in \Gamma\}, \Gamma \vdash_{\mathbf{Q}} A$ iff $\Gamma^{\triangleright} \vdash_{\mathbf{T}^{m}}$ $\triangleright B_{1} \wedge \ldots \wedge \triangleright B_{n}$.

Note that every minimal $D a b$-formula that is $\mathbf{T}$-derivable from $\Gamma^{\triangleright}$ comprises only one disjunct. ${ }^{9}$ This means that, for statements of the form $\Gamma^{\triangleright} \vdash \mathbf{T}^{m}$ $\triangleright B_{1} \wedge \ldots \wedge \triangleright B_{n}$, Minimal Abnormality and Reliability boil down to the Simple strategy. Thus the marking definition may be simplified to: a line is marked iff some member of its condition has been derived on the empty condition. Similarly, a $\mathbf{T}$-model $M$ of $\Gamma^{\triangleright}$ is a $\mathbf{T}^{m}$-model of $\Gamma^{\triangleright}$ iff $A b(M)=\bigcap\left\{A b\left(M^{\prime}\right) \mid M^{\prime}\right.$ is a $\mathbf{T}$-model of $\left.\Gamma^{\triangleright}\right\}=\left\{A \in \Omega \mid \Gamma^{\triangleright} \vdash_{\mathbf{T}} A\right\}$.

Let us now turn to the fact that $\mathrm{C} 2$ does not hold for $\mathbf{Q}$ - for example $\mathbf{P}$-equivalence does not warrant $\mathbf{Q}$-equivalence - whereas $\mathrm{C} 2$ holds for $\mathbf{T}^{m}$ in view of Theorem 7. This opposition obviously derives from the fact that $\mathbf{T}^{m}$ distinguishes between $\triangleright(p \wedge \neg p)$, which has no T-models, and $\triangleright p \wedge \triangleright \neg p$, which

\footnotetext{
${ }^{8}$ Except that, in order to define $\Gamma \vDash_{\mathbf{T}} A$, a $\mathbf{T}$-model is defined as $M=\left\langle W, w_{0}, R, v\right\rangle$ with $w_{0} \in W$ and $M$ is said to verify $A$ iff $v_{M}\left(A, w_{0}\right)=1$.

${ }^{9}$ The property does not hold for all premise sets but is typical for premise sets $\Gamma^{\triangleright}$ with $\Gamma$ a set of modal-free formulas.
} 
does, whereas $\mathbf{Q}$ blurs this distinction. For example $\{p, \neg p\}$ is $\mathbf{P}$-equivalent to $\{p \wedge \neg p\}$, whereas $\{\triangleright p, \triangleright \neg p\}$ is not $\mathbf{T}$-equivalent to $\{\triangleright(p \wedge \neg p)\}$.

The situation is similar for the Strong and Weak consequence relations, which were employed to illustrate the non-applicability of $\mathrm{C} 1$ and $\mathrm{C} 3$. Here we only consider the Strong consequence relation. Let the premises be formulated

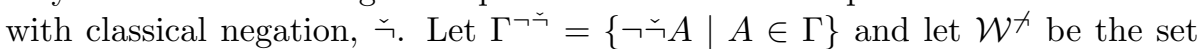
of closed formulas that do not contain $\neg$ (but may contain $\check{\neg}$ ). It was proved in $[5]^{10}$ that $C n_{\text {Strong }}(\Gamma)=C n_{\mathbf{C L u N}^{m}}\left(\Gamma^{\neg \frown}\right) \cap \mathcal{W}^{\rtimes}$. Note that Theorems 1-3 hold for $C n_{\mathbf{C L u N}^{m}}(\Gamma)$, but that they do not hold for the consequence relation

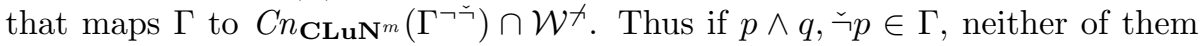
will be in $C n_{\text {Strong }}(\Gamma) ; \neg \check{\neg}(p \wedge q)$ and $\neg \check{\neg} \neg p$ will be members of both $\Gamma \neg \check{\neg}$ and $\left.C n_{\mathbf{C L u N}^{m}}(\Gamma\urcorner \check{\urcorner}\right)$ but obviously not of $\left.C n_{\mathbf{C L u N}^{m}}(\Gamma\urcorner \check{\imath}\right) \cap \mathcal{W}^{\rtimes}$. Note also that the required translation does not complicate the applicability of $\mathrm{C} 1-\mathrm{C} 3$.

\section{Extension of Equivalent Premise Sets}

Let us now turn to the announced related problem: the equivalence of extensions of equivalent premise sets. In this respect adaptive logics do not behave like Tarski logics. At first sight, they seem to behave just as strangely as other formal approaches to DRF.

Fact 1 If $\mathbf{L}$ is a Tarski logic, then $C n_{\mathbf{L}}\left(\Gamma_{1}\right)=C n_{\mathbf{L}}\left(\Gamma_{2}\right)$ warrants that $C n_{\mathbf{L}}\left(\Gamma_{1} \cup\right.$ $\Delta)=C n_{\mathbf{L}}\left(\Gamma_{2} \cup \Delta\right)$.

Fact $2 C n_{\mathbf{A L}}\left(\Gamma_{1}\right)=C n_{\mathbf{A L}}\left(\Gamma_{2}\right)$ does not warrant that $C n_{\mathbf{A L}}\left(\Gamma_{1} \cup \Delta\right)=C n_{\mathbf{A L}}\left(\Gamma_{2} \cup\right.$ $\Delta)$.

That the second fact holds follows immediately from the following example:

$$
\begin{gathered}
C n_{\mathbf{C L u N}^{m}}(\{p\})=C n_{\mathbf{C L u N}^{m}}(\{p \vee(q \wedge \neg q)\}) \text { but } \\
C n_{\mathbf{C L u N}^{m}}(\{p, q \wedge \neg q\}) \neq C n_{\mathbf{C L u N}^{m}}(\{p \vee(q \wedge \neg q), q \wedge \neg q\}) .
\end{gathered}
$$

Note that the example may be adjusted to any adaptive logic in which classical disjunction is present or definable. The example clearly indicates the most straightforward reason why the fact holds. The formula $q \wedge \neg q$ is an abnormality and hence is supposed to be false 'unless and until proven otherwise'. The original premise sets are equivalent because $p \vee(q \wedge \neg q)$ is the only premise of the second premise set and its minimal abnormal interpretation leads to $p$. If, however, $q \wedge \neg q$ is added to the premise sets, $\{p, q \wedge \neg q\}$ still gives us $p$ because $\mathbf{C L u N}^{m}$ is reflexive, but $p$ is not derivable from $\{p \vee(q \wedge \neg q), q \wedge \neg q\}$ because this extended premise set requires $q \wedge \neg q$ to be true and has the same $\mathbf{C L u N}^{m}$ consequences as $\{q \wedge \neg q\}$. To the negative fact corresponds a positive result which is very similar to it.

Theorem 8 If $\mathbf{L}$ is a Tarski logic weaker than $\mathbf{A L}$ and $C n_{\mathbf{L}}\left(\Gamma_{1}\right)=C n_{\mathbf{L}}\left(\Gamma_{2}\right)$, then $C n_{\mathbf{A L}}\left(\Gamma_{1} \cup \Delta\right)=C n_{\mathbf{A L}}\left(\Gamma_{2} \cup \Delta\right)$ for all $\Delta$.

\footnotetext{
${ }^{10}$ The paraconsistent negation is there written as $\sim($ here as $\neg$ ) and the classical negation as $\neg($ here as $\check{\neg})$.
} 
Proof. Suppose that the antecedent is true. In view of the reflexivity of $\mathbf{L}$, (i) $\Delta \subseteq C n_{\mathbf{L}}\left(\Gamma_{1} \cup \Delta\right)$ and (ii) $C n_{\mathbf{L}}\left(\Gamma_{1}\right)=C n_{\mathbf{L}}\left(\Gamma_{2}\right)$ warrants that $\Gamma_{2} \subseteq C n_{\mathbf{L}}\left(\Gamma_{1}\right)$. As $\mathbf{L}$ is monotonic, it follows that $\Gamma_{2} \cup \Delta \subseteq C n_{\mathbf{L}}\left(\Gamma_{1} \cup \Delta\right)$. So $\Gamma_{2} \cup \Delta \subseteq$ $C n_{\mathbf{A L}}\left(\Gamma_{1} \cup \Delta\right)$ in view of $C n_{\mathbf{L}}\left(\Gamma_{1} \cup \Delta\right) \subseteq C n_{\mathbf{A L}}\left(\Gamma_{1} \cup \Delta\right)$. By the same reasoning $\Gamma_{1} \cup \Delta \subseteq C n_{\mathbf{A L}}\left(\Gamma_{2} \cup \Delta\right)$. But then, in view of Theorem 6, $C n_{\mathbf{A L}}\left(\Gamma_{1} \cup \Delta\right)=$ $C n_{\mathbf{A L}}\left(\Gamma_{2} \cup \Delta\right)$.

For adaptive logics there is a weaker alternative for Fact 1. For this, we need another definition.

Definition 10 A set of formulas $\Theta$ is an AL-monotonic extension of a set of formulas $\Gamma$ iff $\Gamma \subset \Theta$ and $C n_{\mathbf{A L}}(\Gamma) \subseteq C n_{\mathbf{A L}}(\Theta)$.

Theorem 9 If $\Gamma_{1} \cup \Delta$ is an $\mathbf{A L}-m o n o t o n i c$ extension of $\Gamma_{1}$ and $\Gamma_{2} \cup \Delta$ is an AL-monotonic extension of $\Gamma_{2}$, then $C n_{\mathbf{A L}}\left(\Gamma_{1}\right)=C n_{\mathbf{A L}}\left(\Gamma_{2}\right)$ warrants that $C n_{\mathbf{A L}}\left(\Gamma_{1} \cup \Delta\right)=C n_{\mathbf{A L}}\left(\Gamma_{2} \cup \Delta\right)$

Proof. Suppose $C n_{\mathbf{A L}}\left(\Gamma_{1}\right)=C n_{\mathbf{A L}}\left(\Gamma_{2}\right), \Gamma_{1} \cup \Delta$ is an AL-monotonic extension of $\Gamma_{1}$ and $\Gamma_{2} \cup \Delta$ is an AL-monotonic extension of $\Gamma_{2}$. By definition 10, the second supposition implies that

$$
C n_{\mathbf{A L}}\left(\Gamma_{1}\right) \subseteq C n_{\mathbf{A L}}\left(\Gamma_{1} \cup \Delta\right)
$$

In view of the reflexivity of adaptive logics

$$
\Delta \subseteq C n_{\mathrm{AL}}\left(\Gamma_{1} \cup \Delta\right) \text {. }
$$

From the two previous results, one obtains immediately that

$$
C n_{\mathbf{A L}}\left(\Gamma_{1}\right) \cup \Delta \subseteq C n_{\mathbf{A L}}\left(\Gamma_{1} \cup \Delta\right),
$$

and with Theorem 3

$$
C n_{\mathbf{A L}}\left(\Gamma_{1} \cup \Delta\right)=C n_{\mathbf{A L}}\left(C n_{\mathbf{A L}}\left(\Gamma_{1}\right) \cup \Delta \cup \Gamma_{1}\right) .
$$

In view of the reflexivity of $\mathbf{A L}, \Gamma_{1} \subseteq C n_{\mathbf{A L}}\left(\Gamma_{1}\right)$, whence:

$$
C n_{\mathbf{A L}}\left(\Gamma_{1} \cup \Delta\right)=C n_{\mathbf{A L}}\left(C n_{\mathbf{A L}}\left(\Gamma_{1}\right) \cup \Delta\right) .
$$

With the same reasoning, the following is provable

$$
C n_{\mathbf{A L}}\left(\Gamma_{2} \cup \Delta\right)=C n_{\mathbf{A L}}\left(C n_{\mathbf{A L}}\left(\Gamma_{2}\right) \cup \Delta\right) .
$$

The supposition implies that $C n_{\mathbf{A L}}\left(C n_{\mathbf{A L}}\left(\Gamma_{1}\right) \cup \Delta\right)=C n_{\mathbf{A L}}\left(C n_{\mathbf{A L}}\left(\Gamma_{2}\right) \cup \Delta\right)$, whence

$$
C n_{\mathbf{A L}}\left(\Gamma_{1} \cup \Delta\right)=C n_{\mathbf{A L}}\left(\Gamma_{2} \cup \Delta\right)
$$

There are criteria for deciding whether an extension is AL-monotonic. The criteria depend on the strategy, which is the third element of the adaptive logic AL. The criteria we introduce below may be not the sharpest possible ones, but it is obvious that they are correct. Let $\Gamma$ be the original premise set and $\Gamma^{\prime}$ the extended premise set. 
For the Reliability strategy, the criterium reads: If $\Gamma \subseteq \Gamma^{\prime}$ and $U\left(\Gamma^{\prime}\right) \subseteq U(\Gamma)$ then $\Gamma^{\prime}$ is an AL-monotonic extension of $\Gamma$. In words: if every abnormality that is unreliable with respect to $\Gamma^{\prime}$ is also unreliable with respect to $\Gamma$, then $\Gamma^{\prime}$ is an AL-monotonic extension of $\Gamma$. In terms of the proof theory, this means that every unmarked line in a proof from $\Gamma$ remains unmarked if the premise set is extended to $\Gamma^{\prime}$. This warrants that the final consequences of $\Gamma$ are also final consequences of $\Gamma^{\prime}$. Obviously, some lines that are marked in a proof from $\Gamma$ may be unmarked in a proof from $\Gamma^{\prime}$. The effect of this is that the latter premise set has more, but not less, consequences than the former.

For the Minimal Abnormality strategy, the criterium reads: If $\Gamma \subseteq \Gamma^{\prime}$ and for every $\Delta^{\prime} \in \Phi\left(\Gamma^{\prime}\right)$, there is a $\Delta \supseteq \Delta^{\prime}$ such that $\Delta \in \Phi(\Gamma)$, then $\Gamma^{\prime}$ is an AL-monotonic extension of $\Gamma$. This criterium is most easily understood from a semantic point of view. The antecedent warrants that every AL-model of $\Gamma^{\prime}$ is an AL-model of $\Gamma$ and hence verifies every formula verified by all AL-models of $\Gamma$.

It is instructive to illustrate the difference between the two criteria in terms of $\mathbf{C L u N}^{r}$ and $\mathbf{C L u N}{ }^{m}$. Let $\Gamma=\{(p \wedge \neg p) \vee(q \wedge \neg q),(p \wedge \neg p) \vee(r \wedge \neg r)$, $s \vee(p \wedge \neg p), s \vee(q \wedge \neg q)\}$ and let $\Gamma^{\prime}=\Gamma \cup\{q \wedge \neg q\}$. As $U(\Gamma)=U\left(\Gamma^{\prime}\right)=\{p \wedge \neg p$, $q \wedge \neg q, r \wedge \neg r\}, \Gamma^{\prime}$ is a $\mathbf{C L u N}^{r}$-monotonic extension of $\Gamma$. Note, however, that $\Phi(\Gamma)=\{\{p \wedge \neg p\},\{q \wedge \neg q, r \wedge \neg r\}\}$ whereas $\Phi\left(\Gamma^{\prime}\right)=\{\{q \wedge \neg q, p \wedge \neg p\},\{q \wedge$ $\neg q, r \wedge \neg r\}\}$. So $\Gamma^{\prime}$ is not a $\mathbf{C L u N}^{m}$-monotonic extension of $\Gamma$ and actually $\Gamma \vdash_{\mathbf{C L u N}^{m}} s$ whereas $\Gamma^{\prime} \nvdash_{\mathbf{C L u N}^{m}} s$.

\section{Maximality of the Lower Limit Logic}

As LLL is a Tarski logic weaker than AL, Theorem 8 entails the following.

Corollary 1 If $C n_{\mathbf{L L L}}\left(\Gamma_{1}\right)=C n_{\mathbf{L L L}}\left(\Gamma_{2}\right)$, then $C n_{\mathbf{A L}}\left(\Gamma_{1} \cup \Delta\right)=C n_{\mathbf{A L}}\left(\Gamma_{2} \cup \Delta\right)$ for all $\Delta$.

Obviously, the corollary can also be proved directly. As LLL is a Tarski logic, $C n_{\mathbf{L L L}}\left(\Gamma_{1}\right)=C n_{\mathbf{L L} \mathbf{L}}\left(\Gamma_{2}\right)$ warrants that $C n_{\mathbf{L L L}}\left(\Gamma_{1} \cup \Delta\right)=C n_{\mathbf{L L L}}\left(\Gamma_{2} \cup \Delta\right)$ by Fact 1 and $C n_{\mathbf{L L L}}\left(\Gamma_{1} \cup \Delta\right)=C n_{\mathbf{L L L}}\left(\Gamma_{2} \cup \Delta\right)$ warrants $C n_{\mathbf{A L}}\left(\Gamma_{1} \cup \Delta\right)=$ $C n_{\mathrm{AL}}\left(\Gamma_{2} \cup \Delta\right)$ by Theorem 7 .

We shall now prove that the lower limit logic LLL of an adaptive logic AL is not only a Tarski logic that is weaker than AL but that actually every monotonic logic $\mathbf{L}$ that is weaker than $\mathbf{A L}$ is weaker than $\mathbf{L L L}$ or identical to LLL. In the proof of the following theorem we rely on the compactness of $\mathbf{L L L}$, but do not require $\mathbf{L}$ to be compact.

Theorem 10 For all monotonic logics $\mathbf{L}$ weaker than $\mathbf{A L}^{m}$ and for all $\Gamma$, $C n_{\mathbf{L}}(\Gamma) \subseteq C n_{\mathbf{L L L}}(\Gamma)$.

Proof. Suppose that $\mathbf{L}$ is a monotonic logic weaker than $\mathbf{A} \mathbf{L}^{m}$ and that there is a $\Gamma$ and a $B$ for which the following three hold.

$$
\begin{gathered}
\Gamma \nvdash_{\mathbf{L L L}} B \\
\Gamma \vdash_{\mathbf{L}} B \\
\Gamma \vdash_{\mathbf{A L}^{m}} B
\end{gathered}
$$


Let $\Gamma^{\prime}=\left\{\operatorname{Dab}(\Delta) \mid \Gamma \vdash_{\mathbf{L L L}} B \check{V} \operatorname{Dab}(\Delta)\right\}$. In view of the definition of $\Gamma^{\prime},(1)$ entails (4); (5) follows from (2) by the monotonicity of $\mathbf{L}$, and (6) follows from (5) by the supposition.

$$
\begin{gathered}
\Gamma \cup \Gamma^{\prime} \nvdash_{\mathbf{L L L}} B \\
\Gamma \cup \Gamma^{\prime} \vdash_{\mathbf{L}} B \\
\Gamma \cup \Gamma^{\prime} \vdash_{\mathbf{A L}^{m}} B
\end{gathered}
$$

In view of Theorem 4, it follows from (6) and (4) that, for every $\varphi \in \Phi\left(\Gamma \cup \Gamma^{\prime}\right)$, there is a $\Delta \subset \Omega$ such that

$$
\Gamma \cup \Gamma^{\prime} \vdash_{\mathbf{L L L}} B \check{\vee} \operatorname{Dab}(\Delta), \Delta \neq \emptyset \text { and } \Delta \cap \varphi=\emptyset .
$$

In view of the compactness and monotonicity of LLL there are $\operatorname{Dab}\left(\Delta_{1}\right), \ldots$, $\operatorname{Dab}\left(\Delta_{n}\right) \in \Gamma^{\prime}$ such that

$$
\Gamma \cup\left\{\operatorname{Dab}\left(\Delta_{1}\right), \ldots, \operatorname{Dab}\left(\Delta_{n}\right)\right\} \vdash_{\mathbf{L L L}} B \check{\vee} \operatorname{Dab}(\Delta) .
$$

As $\Gamma \vdash_{\mathbf{L L L}} B \check{\vee} \operatorname{Dab}\left(\Delta_{i}\right)$ for every $i \in\{1, \ldots, n\}$,

$$
\Gamma \vdash_{\mathbf{L L L}} B \check{\vee}\left(\bigwedge_{i=1}^{n} \operatorname{Dab}\left(\Delta_{i}\right)\right) .
$$

From (8) follows

$$
\Gamma \cup\left\{\check{\bigwedge}_{i=1}^{n} \operatorname{Dab}\left(\Delta_{i}\right)\right\} \vdash_{\mathbf{L L L}} B \check{\vee} \operatorname{Dab}(\Delta),
$$

whence, by the Deduction Theorem,

$$
\Gamma \vdash_{\mathbf{L L L}}\left(\bigwedge_{i=1}^{n} \operatorname{Dab}\left(\Delta_{i}\right)\right) \check{\supset}(B \check{V} \operatorname{Dab}(\Delta)) .
$$

From (9) and (11) follows

$$
\Gamma \vdash_{\mathbf{L L L}} B \check{\vee} \operatorname{Dab}(\Delta),
$$

whence $\operatorname{Dab}(\Delta) \in \Gamma^{\prime}$. But then every $\varphi \in \Phi\left(\Gamma \cup \Gamma^{\prime}\right)$ contains at least one member of $\Delta$, which contradicts $(7)$.

It follows from Theorem 5 that this result also holds when the third element of AL is Reliability. Hence we obtain the following corollary.

Corollary $\mathbf{2}$ Every monotonic logic $\mathbf{L}$ that is weaker than $\mathbf{A L}$ is weaker than LLL or identical to $\mathbf{L L L}$.

Lemma 1 gives us a further corollary.

Corollary $\mathbf{3}$ If $C n_{\mathbf{A L}}(\Gamma)$ is closed under a monotonic logic $\mathbf{L}$, then $\mathbf{L}$ is weaker than LLL or identical to LLL.

The upshot is that the lower limit logic LLL allows for very sharp applications of $\mathrm{C} 2$ and C3. Moreover, the lower limit logic is the strongest Tarski logic $\mathbf{L}$ weaker than $\mathbf{A L}$ for which holds: if two premise sets are $\mathbf{L}$-equivalent and both are extended with the same set of formulas, then these extensions are AL-equivalent. All this highlights the pivotal role of the lower limit logic. 


\section{In Conclusion}

We have proved that criteria $\mathrm{C} 1-\mathrm{C} 3$, which are standard for identifying equivalent premise sets with respect to Tarski logics, also apply to adaptive logics. This is a major advantage of adaptive logics in comparison to other formal approaches to defeasible reasoning forms because the criteria are transparent and easy to check. This is especially so in view of the pivotal role played by the lower limit logic.

With respect to extensions of equivalent premise sets, adaptive logics do not behave like Tarski logics, but we have located a criterion that is simple and close to that for Tarski logics - and moreover a criterion that is specific for adaptive logics. We have also shown that the strongest Tarski logic weaker than an adaptive logic in standard format is its lower limit and that the lower limit logic is the strongest Tarski logic under which the adaptive consequence set is closed. This means that equivalence with respect to the lower limit logic does not only provide a criterion for adaptive equivalence, but also provides a maximally strong criterion for deciding that similar extensions of adaptively equivalent premise sets are adaptively equivalent.

\section{References}

[1] Atocha Aliseda. Abductive Reasoning. Logical Investigations into Discovery and Explanation. Springer, Dordrecht, 2006.

[2] Grigoris Antoniou. Nonmonotonic Reasoning. MIT Press, Cambridge, Mass., 1996.

[3] Diderik Batens. Inconsistency-adaptive logics and the foundation of nonmonotonic logics. Logique et Analyse, 145:57-94, 1994. Appeared 1996.

[4] Diderik Batens. Inconsistency-adaptive logics. In Orłowska [41], pages $445-472$.

[5] Diderik Batens. Towards the unification of inconsistency handling mechanisms. Logic and Logical Philosophy, 8:5-31, 2000. Appeared 2002.

[6] Diderik Batens. A general characterization of adaptive logics. Logique et Analyse, 173-175:45-68, 2001. Appeared 2003.

[7] Diderik Batens. A strengthening of the Rescher-Manor consequence relations. Logique et Analyse, 183-184:289-313, 2003. Appeared 2005.

[8] Diderik Batens. A universal logic approach to adaptive logics. Logica Universalis, 1:221-242, 2007.

[9] Diderik Batens. Towards a dialogic interpretation of dynamic proofs. In Cédric Dégremont, Laurent Keiff, and Helhe Rückert, editors, Dialogues, Logics and Other Strange Things. Essays in Honour of Shahid Rahman. College Publications, London, 2009 (in print).

[10] Diderik Batens, Joke Meheus, and Dagmar Provijn. An adaptive characterization of signed systems for paraconsistent reasoning. To appear. 
[11] Diderik Batens, Joke Meheus, Dagmar Provijn, and Liza Verhoeven. Some adaptive logics for diagnosis. Logic and Logical Philosophy, 11/12:39-65, 2003.

[12] Diderik Batens and Timothy Vermeir. Direct dynamic proofs for the Rescher-Manor consequence relations: The flat case. Journal of Applied Non-Classical Logics, 12:63-84, 2002.

[13] Salem Benferhat, Didier Dubois, and Henri Prade. Some syntactic approaches to the handling of inconsistent knowledge bases: A comparative study. Part 1: The flat case. Studia Logica, 58:17-45, 1997.

[14] Salem Benferhat, Didier Dubois, and Henri Prade. Some syntactic approaches to the handling of inconsistent knowledge bases: A comparative study. Part 2: The prioritized case. In Orłowska [41], pages 473-511.

[15] Philippe Besnard and Torsten Schaub. Signed systems for paraconsistent reasoning. Journal of Automated Reasoning, 20:191-213, 1998.

[16] Gerhard Brewka. Nonmonotonic Reasoning: Logical Foundations of Commonsense. Cambridge University Press, Cambridge, Mass., 1991.

[17] Newton C.A. da Costa, Otávio Bueno, and Steven French. The logic of pragmatic truth. Journal of Philosophical Logic, 27:603-620, 1998.

[18] Kristof De Clercq. Two new strategies for inconsistency-adaptive logics. Logic and Logical Philosophy, 8:65-80, 2000. Appeared 2002.

[19] Phan Minh Dung. On the acceptability of arguments and its fundamental role in nonmonotonic reasoning, logic programming and n-person games. Artificial Intelligence, 77:321-358, 1995.

[20] Lou Goble. A proposal for dealing with deontic dilemmas. In Alessio Lomuscio and Donald Nute, editors, Deontic Logic in Computer Science, Proceedings of DEON 2004, volume 3065 of Lecture Notes in Computer Science, pages 74-113, Berlin, 2004. Springer.

[21] Lou Goble. A logic for deontic dilemmas. Journal of Applied Logic, 3:461483, 2005.

[22] G.E. Hughes and M.J. Cresswell. An Introduction to Modal Logic. Methuen, London, New York, 1972. First published 1968.

[23] Allen L. Brown Jr. and Yoav Shoham. New results on semantical nonmonotonic reasoning. In Michael Reinfrank, Johan de Kleer, Matthew L. Ginsberg, and Erik Sandewall, editors, NMR, volume 346 of Lecture Notes in Computer Science, pages 19-26. Springer, 1988.

[24] Sébastien Konieczny and Ramón Pino Pérez. Merging information under constraints: A logical framework. Journal of Logic and Computation, 12:773-808, 2002.

[25] Theo A. F. Kuipers. From Instrumentalism to Constructive Realism. On some Relations Between Confirmation, Empirical Progress, and Truth Approximation, volume 287 of Synthese Library. Kluwer, Dordrecht, 2000. 
[26] Daniel Lehmann and Menachem Magidor. What does a conditional knowledge base entail? Artificial Intelligence, 55(1):1-60, 1992.

[27] Bert Leuridan. Causal discovery and the problem of ignorance. an adaptive logic approach. Journal of Applied Logic, to appear.

[28] Fangzhen Lin and Yoav Shoham. Epistemic semantics for fixed-points nonmonotonic logics. In TARK '90: Proceedings of the 3rd conference on Theoretical aspects of reasoning about knowledge, pages 111-120, San Francisco, CA, USA, 1990. Morgan Kaufmann Publishers Inc.

[29] Witold Łukaszewicz. Non-Monotonic Reasoning. Formalization of Commonsense Reasoning. Ellis Horwood, New York, 1990.

[30] Lorenzo Magnani, Nancy J. Nersessian, and Claudio Pizzi, editors. Logical and Computational Aspects of Model-Based Reasoning. Kluwer, Dordrecht, 2002.

[31] David Makinson. Bridges from Classical to Nonmonotonic Logic, volume 5 of Texts in Computing. King's College Publications, London, 2005.

[32] Joke Meheus. Erotetic arguments from inconsistent premises. Logique et Analyse, 165-166:49-80, 1999. Appeared 2002.

[33] Joke Meheus. Adaptive logics for question evocation. Logique et Analyse, 173-175:135-164, 2001. Appeared 2003.

[34] Joke Meheus. An adaptive logic for pragmatic truth. In Walter A. Carnielli, Marcelo E. Coniglio, and Itala M. Loffredo D'Ottaviano, editors, Paraconsistency. The Logical Way to the Inconsistent, pages 167-185. Marcel Dekker, New York, 2002.

[35] Joke Meheus. Empirical progress and ampliative adaptive logics. In Roberto Festa, Atocha Aliseda, and Jeanne Peijnenburg, editors, Confirmation, Empirical Progress, and Truth Approximation. Essays in Debate with Theo Kuipers. Vol. 1, volume 83 of Poznan Studies in the Philosophy of the Sciences and the Humanities, pages 193-217. Rodopi, Amsterdam/New York, 2005.

[36] Joke Meheus and Diderik Batens. A formal logic for abductive reasoning. Logic Journal of the IGPL, 14:221-236, 2006.

[37] Joke Meheus and Dagmar Provijn. Abduction through semantic tableaux versus abduction through goal-directed proofs. Theoria, 22/3(60):295-304, 2007.

[38] Joke Meheus and Christian Straßer. Avoiding deontic explosion by contextually restricting modal inheritance. forthcoming.

[39] Joke Meheus, Liza Verhoeven, Maarten Van Dyck, and Dagmar Provijn. Ampliative adaptive logics and the foundation of logic-based approaches to abduction. In Magnani et al. [30], pages 39-71. 
[40] Irene Mikenberg, Newton C. A. da Costa, and Rolando Chuaqui. Pragmatic truth and approximation to truth. Journal of Symbolic Logic, 51:201-221, 1986.

[41] Ewa Orłowska, editor. Logic at Work. Essays Dedicated to the Memory of Helena Rasiowa. Physica Verlag (Springer), Heidelberg, New York, 1999.

[42] Judea Pearl. Causality. Models, Reasoning, and Inference. Cambridge University Press, Cambridge, 2000.

[43] Giuseppe Primiero and Joke Meheus. Majority merging by adaptive counting. Synthese, 165:203-223, 2008.

[44] Dagmar Provijn and Erik Weber. Adaptive logics for non-explanatory and explanatory diagnostic reasoning. In Magnani et al. [30], pages 117-142.

[45] Raymond Reiter. A theory of diagnosis from first principles. Artificial Intelligence, 32:57-95, 1987.

[46] Nicholas Rescher and Ruth Manor. On inference from inconsistent premises. Theory and Decision, 1:179-217, 1970.

[47] Peter K. Schotch and Raymond E. Jennings. On detonating. In Graham Priest, Richard Routley, and Jean Norman, editors, Paraconsistent Logic. Essays on the Inconsistent, pages 306-327. Philosophia Verlag, München, 1989.

[48] Yoav Shoham. A semantical approach to nonmonotic logics. In $L I C S$, pages 275-279. IEEE Computer Society, 1987.

[49] Christian Straßer. An adaptive logic for rational closure. Forthcoming.

[50] Christian Straßer. An adaptive logic framework for conditional obligations and deontic dilemmas. Logic and Logical Philosophy, 200x. Forthcoming.

[51] Christian Straßer and Dunja Šešelja. Towards the proof-theoretic unification of dung's argumentation framework: An adaptive logic approach. Forthcoming.

[52] Maarten Van Dyck. Causal discovery using adaptive logics. Towards a more realistic heuristics for human causal learning. Logique et Analyse, 185-188:5-32, 2004. Appeared 2005.

[53] Peter Verdée and Stephan van der Waart van Gulik. A generic framework for adaptive vague logics. Studia Logica, 90:385-405, 2008.

[54] Liza Verhoeven. Proof theories for some prioritized consequence relations. Logique et Analyse, 183-184:325-344, 2003. appeared 2005.

[55] Andrzej Wiśniewski. The Posing of Questions. Logical Foundations of Erotetic Inferences. Kluwer, Dordrecht, 1995. 